Journal of Computer Science 6 (11): 1233-1236, 2010

ISSN 1549-3636

(C) 2010 Science Publications

\title{
Development of Fuzzy Controller for Water Level in Stream Boiler Tank
}

\author{
Surachai Panich \\ Srinakharinwirot University, 114 Sukhumvit 23, Bangkok 10110, Thailand
}

\begin{abstract}
Problem statement: The process control of steam boiler is very popular used in the industrial. The temperature of the water is transferred directly by electrical heater. The pressure will increase based on the changing of the temperature. The purpose of the control is to change the opening set point for the valve when the temperature and pressure in the tank are changed. For this problem, we develop fuzzy algorithm to adjust the optimal percentage of valve open. Approach: In this study, the fuzzy control application was programmed in fuzzy control language in form of the function block using structure control language. The input information consisted of real variables in the form of measurable process variables, as well as set points. And the output variables were real variables in the form of correcting variables. Results: The fuzzy control was developed, which consists of two input variables, the degree of temperature and pressure in boiler tank measured by sensor. For fuzzy system of water level control, the algorithm is basically implemented in form of the MATLAB code. In the experiment, we assumed that the water level would not effect to the temperature and pressure. Conclusion: The research for the development of the fuzzy logic and the model was tested with the step inputs and the changing of the inputs. The whole simulation process was built to test the behavior of the system when the inputs change.
\end{abstract}

Key words: Fuzzy algorithm, steam boiler tank

\section{INTRODUCTION}

In recent years, fuzzy logic has proven well its broad potential in industrial automation applications. In this application area, engineers primarily rely on proven concepts. Traditional system modeling and analysis techniques are too precise for such problems and in order to make complexity less daunting we introduce appropriate simple to achieve a satisfactory compromise between the information we have and the amount of uncertainty we are willing to accept. In this aspect, fuzzy systems theory is similar to other engineering theories, because almost all of them characterize the real world in an approximate manner. The Fuzzy Logic tool was introduced in 1965, also by Lotfi Zadeh and is a mathematical tool for dealing with uncertainty. Zadeh, originally an engineer and systems scientist, was concerned with the rapid decline in information afforded by traditional mathematical models as the complexity of the target system increased. Real world problems are too complex and the complexity involves the degree of uncertainty as uncertainty increases, so does the complexity of the problem (Figliola and Beasley, 2005). It offers to a soft computing partnership the important concept of computing with words. It provides a technique to deal with imprecision and information granularity. The fuzzy theory provides a mechanism for representing linguistic constructs such as "many," "low," "medium," "often," "few." In general, the fuzzy logic provides an inference structure that enables appropriate human reasoning capabilities. On the contrary, the traditional binary set theory describes crisp events, events that either do or do not occur. It uses probability theory to explain if an event will occur, measuring the chance with which a given event is expected to occur. The theory of fuzzy logic is based upon the notion of relative graded membership and so are the functions of mutation and cognitive processes. The utility of fuzzy sets lies in their ability to model uncertain or ambiguous data as shown in Fig. 1, so often encountered in real life. It is important to observe that there is an intimate connection between fuzziness and complexity. As the complexity of a task or of a system for performing that task, exceeds a certain threshold, the system must necessarily become fuzzy in nature.

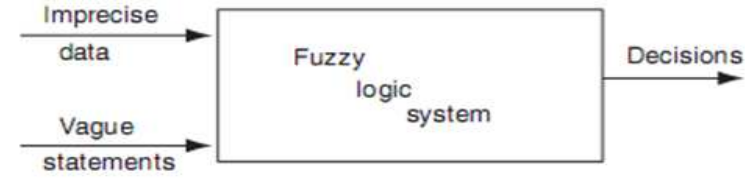

Fig. 1: A fuzzy logic system which accepts imprecise data and vague statements such as low, medium, high and provides decisions 


\section{MATERIALS AND METHODS}

In this study, the fuzzy control application is programmed in fuzzy control language in form of the function block using structure control language. The input information consists of real variables in the form of measurable process variables, as well as set points. And the output variables are real variables in the form of correcting variables. Between the input and output variables of the process, there must be a transformation performing the rule base in form of the fuzzy language. The main components of fuzzy control comprise the linguistic rules base and the inference.

Fuzzification: In this study, there are two input variables to the fuzzy block that are temperature variable and pressure variable. These values must be converted in form of the fuzzy world by the procedure, which is called fuzzification. This procedure needs to do some calculation, which applies the graphic analysis to the input values in the $\mathrm{X}$-axis and their membership degree in Y-axis. There are some different types of membership functions commonly used in practice that are the triangular and trapezoidal membership functions (Sharmeela et al., 2007; Drainkov et al., 1996; Murugavalli and Rajamani, 2007; Tao and Taur, 1995; Fan and Huang, 2009; Wahyudi and Salami, 2007). In this application, the triangular functions were applied because of the simplicity and efficiency of implementation.

Temperature input variable: By using the fuzzy set, the operation of fuzzy controller is to determine the percentage of valve open (v) for each pressure level to control the level of water in stream boiler tank. By using the fuzzy control method, assume that the degree of temperature $(\hat{\mathrm{t}})$ is expressed by a number in the interval $\left[0 t_{\max }\right]$, where $t_{\max }$ is some positive number depend on the chosen measurement unit. The membership functions of temperature consists of five fuzzy logic ranges that can be defined using the linguistic terms as Very Low (VLO), Low (LO), Medium (M), Large (LA) and Very Large (VLA).

Pressure input variable: Again for the level of pressure $(\hat{\mathrm{p}})$ is expressed by a number in the interval $\left[0, p_{\max }\right]$ and divide the amount into five levels, Very Low (VLO), Low (LO), Medium (M), Large (LA) and Very Large (VLA). Then it is reasonable to represent these linguistic terms by the triangular shape fuzzy number known in Fig. 2.

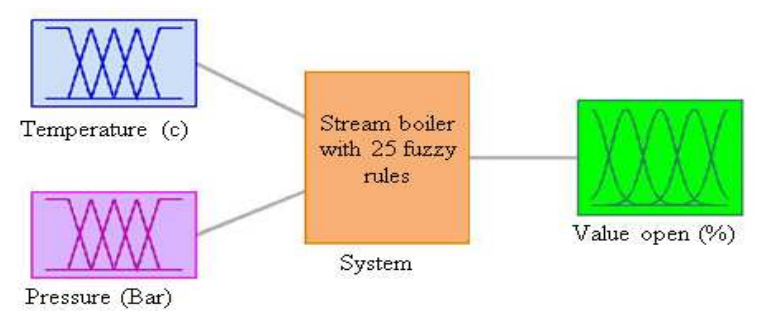

Fig. 2: Fuzzy control for controller

Table 1: Inference rules for fuzzy of valve open

\begin{tabular}{|c|c|c|c|c|c|c|}
\hline \multirow{2}{*}{\multicolumn{2}{|c|}{$\begin{array}{l}\text { (inference rules } \\
\text { for fuzzy for percentage } \\
\text { of valve open) }\end{array}$}} & \multicolumn{5}{|c|}{$\mathrm{p}$ (pressure) } \\
\hline & & VLO & LO & $\mathrm{M}$ & LA & VLA \\
\hline \multirow[t]{5}{*}{ t (temperature) } & VLO & $\mathrm{SC}$ & $\mathrm{SC}$ & $\mathrm{C}$ & M & M \\
\hline & LO & $\mathrm{SC}$ & $\mathrm{SC}$ & $\mathrm{C}$ & M & $\mathrm{M}$ \\
\hline & $\mathrm{M}$ & $\mathrm{SC}$ & $\mathrm{C}$ & $\mathrm{O}$ & $\mathrm{O}$ & $\mathrm{O}$ \\
\hline & LA & $\mathrm{C}$ & M & $\mathrm{O}$ & $\mathrm{SO}$ & SO \\
\hline & VLA & $\mathrm{C}$ & M & $\mathrm{O}$ & $\mathrm{SO}$ & SO \\
\hline
\end{tabular}

Percentage of valve open for output variable: By using fuzzy controllers we can approximate the function with relative ease on the basis of human intuition and experience. To do that, we need to define the linguistic variable representing the required Percentage of valve open by v. We want to control with five distinction characterizing, Strong Close (SC), Close (C), Medium (M), Open (O), Strong Open (SO). The membership functions of pressure consist of five fuzzy logic ranges that can be defined using the linguistic terms as Very Low, Low, Medium, Large and Very Large shown in Table 1.

\section{RESULTS}

For fuzzy system of water level control, the algorithm is basically implemented in form of the MATLAB code. In the simulation, we assume with the condition that the water level will not effect to the temperature and pressure.

Step 1: Determine input variables $t$ and $\mathrm{p}$ compatibility with the corresponding antecedents of all inference rules as shown in Fig. 3 and 4. Only rules for which the compatibilities of the measured values with both antecedents are positive take place in determining the value of the controlled variable $v$ as shown in Fig. 5.

Step 2: Convert the fuzzy set represents the overall conclusion into a real number.

The most common method is to determine the value for which the area under the graph of the membership function is equally divided. This method is called a center of gravity defuzzification method as shown in Fig. 6. 


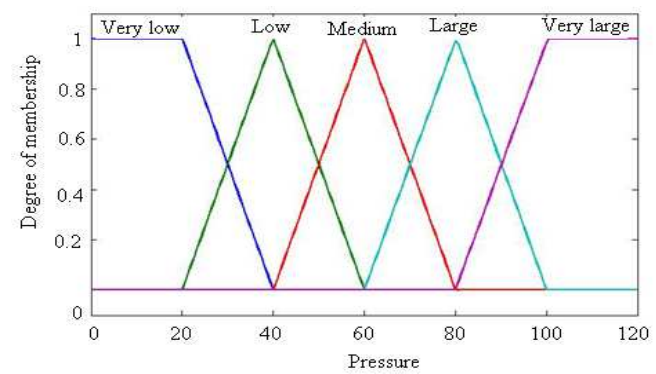

Fig. 3: Fuzzy control for values of temperature (t)

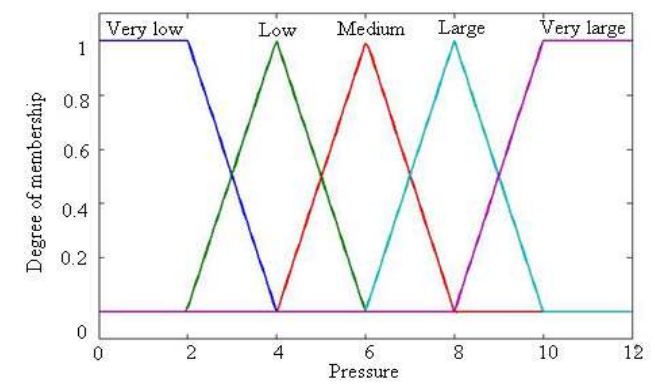

Fig. 4: Fuzzy control for values of pressure (p)

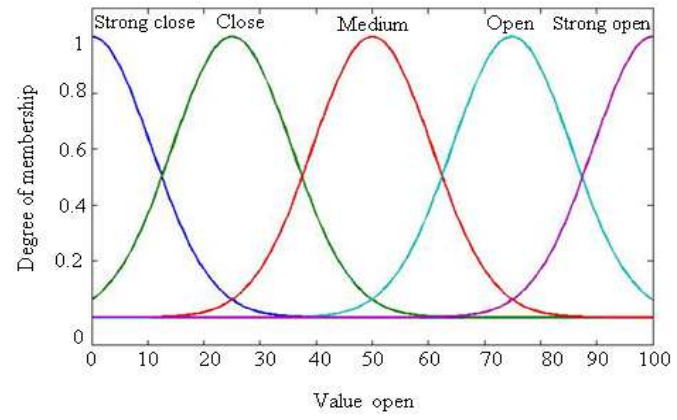

Fig. 5: Fuzzy control for percentage of valve opens (v)

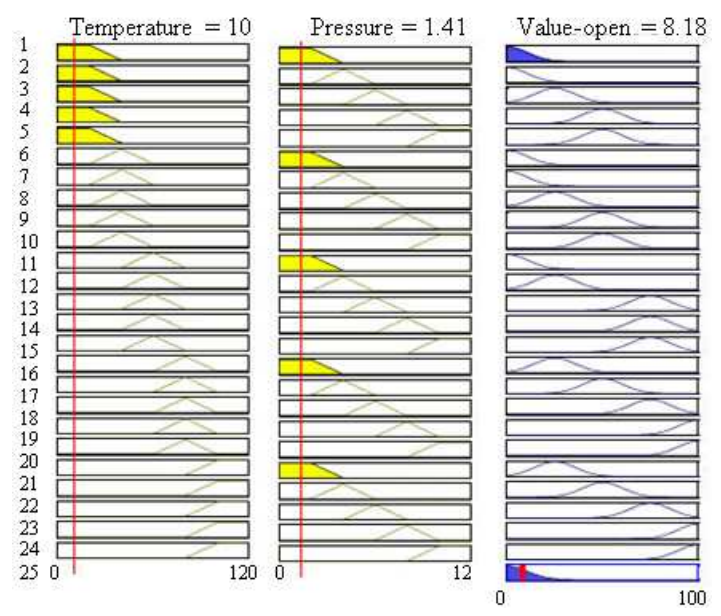

Fig. 6: Center of gravity defuzzification method

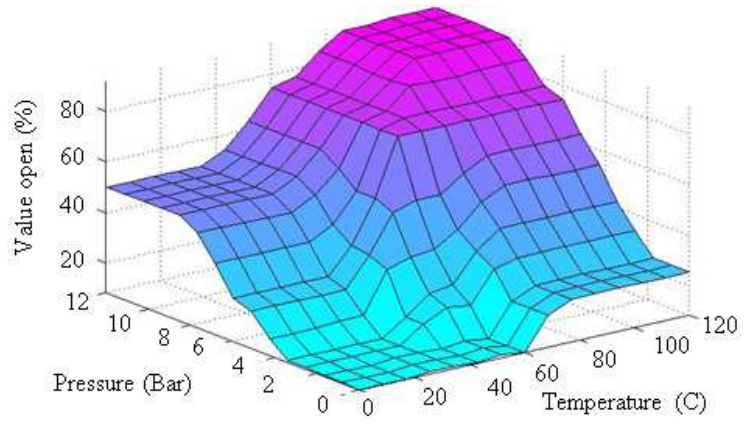

Fig. 7: Result of fuzzy controller for percentage of valve open

Also the result of fuzzy controller for percentage of valve open is generated as shown in Fig. 7.

\section{DISCUSSION}

In this research, the main objective has been achieved. The recommendation for the next development is the PID controller or the neuron network, which use to control valve instead of fuzzy logic algorithm.

\section{CONCLUSION}

The research for the development of the fuzzy logic and the model is tested with the step inputs and the changing of the inputs. The whole simulation process was built to test the behavior of the system when the inputs change. A fuzzy logic controller for temperature, pressure and one fuzzy logic controller for flow and level control are implemented in MATLAB software. The development of the fuzzy logic for the process control is one of the best ways for the automation engineer to built the controller by himself based on his professional and experience in the factory where the nonlinear and the unstable process are present, which are difficult to control by the conventional controller. The benefit of the fuzzy controller is that it is mainly based on the experience of the process engineers. It sometimes can handle the tasks, which were normally implemented by the engineer.

\section{ACKNOWLEDGEMENT}

This research from Measurement and Mobile Robot Laboratory (M and M-LAB) was supported by Faculty of Engineering, Srinakharinwirot University. 


\section{REFERENCES}

Drainkov, D., H. Jellendoorn, M. Reinfrank, L. Ljung and R. Palm et al., 1996. An Introduction to Fuzzy Control. 2nd Edn., Springer, Berlin, ISBN: 10: 3540606912, pp: 316.

Fan, Z. and M. Huang, 2009. Fuzzy rule set based engine fault diagnosis. Proceeding of the AsiaPacific Power and Energy Engineering Conference, Mar. 27-31, IEEE Xplore Press, Wuhan, pp: 1-5. DOI: 10.1109/APPEEC.2009.4918394

Figliola, R.S. and D.E. Beasley, 2005. Theory and Design for Mechanical Measurements. 4th Edn., Wiley, New York, ISBN: 10: 0471445932, pp: 560.

Murugavalli, S. and V. Rajamani, 2007. An improved implementation of brain tumor detection using segmentation based on neuro fuzzy technique. J. Comput. $\quad$ Sci., $\quad 3: \quad 841-846$. http://www.scipub.org/fulltext/jcs/jcs311841-846.pdf
Sharmeela, C., M.R. Mohan, G. Uma and J. Baskaran, 2007. Fuzzy logic controller based three-phase shunt active filter for line harmonics reduction. J. Comput. $\quad$ Sci., $\quad 3: \quad 76-80$. http://www.scipub.org/fulltext/jcs/jcs3276-80.pdf

Tao, C.W. and J.S. Taur, 1995. A robust fuzzy control of a nonlinear magnetic ball suspension system. Proceeding of the 1995. Proceeding of the International EEE/IAS Conference on Industrial Automation and Control: Emerging Technologies, May 22-27, IEEE Xplore Press, Taipei, Taiwan, pp: 365-369. DOI: 10.1109/IACET.1995.527589

Wahyudi, R.M. and M.J.E. Salami, 2007. Fuzzy antiwindup schemes for NCTF control of Point-Topoint (PTP) positioning systems. Am. J. Applied Sci., $\quad 4$ : 220-228. http://www.scipub.org/fulltext/ajas/ajas44220-228.pdf 